\title{
A STATISTICAL ANALYSIS OF THE DANGER INDUCED BY HOM EXCITATION IN A SUPERCONDUCTING LINAC
}

\author{
J-L. Biarrotte, Institut de Physique Nucléaire, Division Accélérateurs, 91406 Orsay Cedex, France \\ biarrott@ipno.in2p3.fr
}

\section{Abstract}

High order modes (HOM) excitation is a serious problem for circular machines where HOM can be excited and enhanced at each turn under resonance conditions. In linear machines, the problem is quite different as the beam passes only once through each cavity. In continuous (CW) operation for example, a HOM may build up only in the very unlucky case where its frequency happens to be exactly a multiple of the bunch frequency. A careful analysis shows that the probability of occurrence of HOM build-up is extremely low in a superconducting (SC) linac running under $\mathrm{CW}$ operation, leading to the conclusion that there is no need for specific couplers. Some elements are also given for pulsed operation.

\section{INTRODUCTION}

The analysis of high order modes excitation in superconducting cavities is a very important issue because this excitation, if resonant, can lead both to significant additional cryogenic losses and to serious beam quality degradations. This is why specific HOM couplers are systematically used in SCRF cavities to damp the most dangerous modes below some safety conditions; but one may ask if this unconditional approach is always relevant...

We try here to quantify the real danger induced by HOM excitation in a SC linac, focusing mainly on powerinduced considerations. This study has been developed within the frame of the French-Italian collaboration (CEA, INFN, IN2P3), aiming at the design of a SC proton linac operating with a $352.2 \mathrm{MHz} \mathrm{CW}$ beam and dedicated to nuclear waste transmutation [1].

\section{HOM EXCITATION BY A CW BEAM}

Let's here consider the case of a high order resonant mode [angular freq.: $\omega$; normalised shunt impedance: $\mathrm{r} / \mathrm{Q}$ - circuit definition; loaded quality factor: $\mathrm{Q}_{\mathrm{L}}$ ] excited by a $\mathrm{CW}$ beam [bunch period: $\mathrm{T}_{\mathrm{b}}$; main current: $\mathrm{I}_{0}$; bunch length: $\sigma_{b}-$ gaussian bunches and $\sigma_{b}<<T_{b}$ assumed]. From the equivalent circuit theory, and given some preliminary assumptions generally fulfilled in superconducting cavities (constant particle speed through the cavity, slow fields decay: $\mathrm{Q}_{\mathrm{L}}>>\omega \mathrm{L} / \beta \mathrm{c}$ ), one can show that the power deposited onto an excited HOM takes the form (see for example [2]):

$$
\mathrm{P}=\frac{1}{2} \omega \mathrm{T}_{\mathrm{b}}\left(\frac{\mathrm{r}}{\mathrm{Q}}\right) \mathrm{I}_{0}^{2} \mathrm{e}^{-\omega^{2} \sigma_{\mathrm{b}}^{2}} \mathrm{~F}\left(\mathrm{~T}_{\mathrm{b}}, \tau_{\text {cav }}\right)
$$

where $\mathrm{F}\left(\mathrm{T}_{\mathrm{b}}, \tau_{\text {cav }}\right)=\frac{1-\mathrm{e}^{-2 \mathrm{~T}_{\mathrm{b}} / \tau_{\text {cav }}}}{1-2 \cos \left(\omega \mathrm{T}_{\mathrm{b}}\right) \mathrm{e}^{-\mathrm{T}_{\mathrm{b}} / \tau_{\text {cav }}}+\mathrm{e}^{-2 \mathrm{~T}_{\mathrm{b}} / \tau_{\text {cav }}}}$ and $\tau_{\text {cav }}=\frac{2 \mathrm{Q}_{\mathrm{L}}}{\omega}$.

In the high damping limit $\left(\tau_{\mathrm{cav}}<<\mathrm{T}_{\mathrm{b}}\right)$, each bunch sees a practically empty cavity, and Eq. 1 reduces to the case of a single bunch since $\mathrm{F}\left(\mathrm{T}_{\mathrm{b}}, \tau_{\text {cav }}\right) \rightarrow 1$.

The usual running of a SC cavity is described by the low damping limit $\left(\mathrm{T}_{\mathrm{b}}<<\tau_{\text {cav }}\right)$ : in this case, a resonant build-up happens when the excited HOM frequency is a multiple of the bunch frequency (see Fig. 1, black curve). Note that Eq. 1 simplifies, both in the "resonant case" and in the "anti-resonant case".

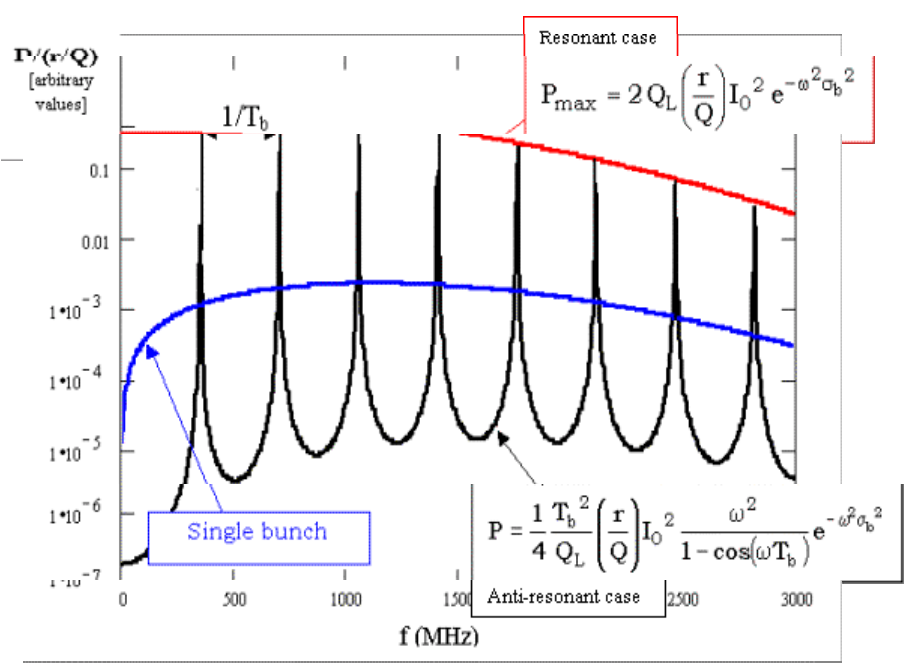

Figure 1: Power deposited onto a HOM as a function of the HOM frequency: case of a single bunch (blue curve) and of a CW beam (black curve - the red curve represents the maximum envelope).

In the low damping limit, one of the most critical parameters is the loaded Q of the excited HOM: the highest the $\mathrm{Q}_{\mathrm{L}}$ is, the highest the deposited power is in the "resonant case", but the lowest it is in the "anti-resonant case", and the narrowest the resonance bandwidth will be (see Fig. 2). In other words, this means that, given a bunch resonance frequency $f_{0}$ :

- If the HOM frequency $f$ is very close to $f_{0}$ (i.e. if $\left|\mathrm{f}-\mathrm{f}_{0}\right|<\mathrm{f}_{0} / 2 \mathrm{Q}_{\mathrm{L}}$ - usually in the range of $10 \mathrm{~Hz}$ to 100 $\mathrm{kHz}$ ), then the HOM will build-up in a resonant way: in this case, the more the mode is damped (i.e. the lowest the $\mathrm{Q}_{\mathrm{L}}$ is), the lower the deposited power will be. 
- If the HOM frequency $f$ is not very close to $f_{0}$, then the HOM will be excited in a non-resonant way: one can show then that there is a value of $\mathrm{Q}_{\mathrm{L}}$ $\left(=\mathrm{f}_{0} / 2\left|\mathrm{f}-\mathrm{f}_{0}\right|\right)$ which maximises the power deposited onto the mode. In this case, the use of a HOM coupler can lead to a worst situation (see Fig. 3).

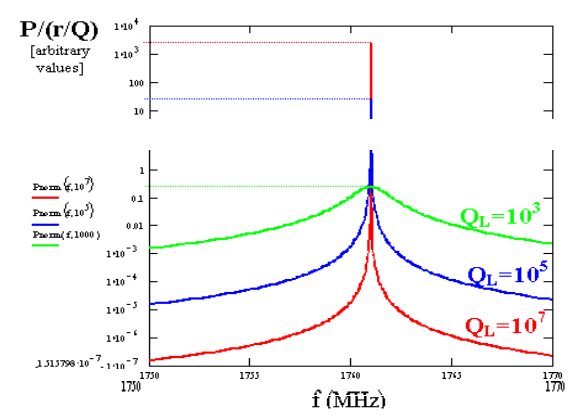

Figure 2: Influence of the loaded Q on the power deposited onto a HOM by a CW beam.

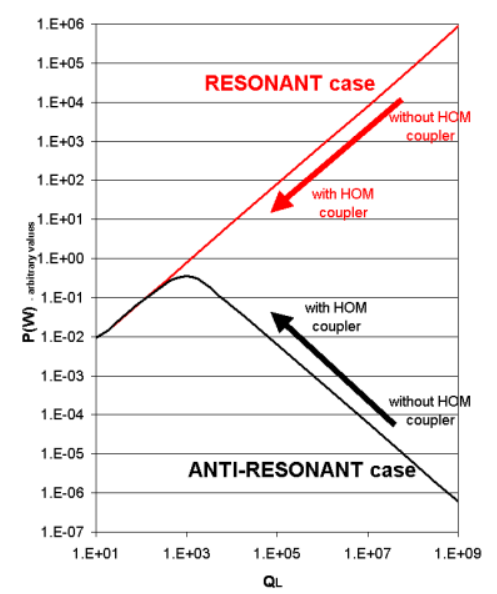

Figure 3: Influence of a HOM coupler on the power deposited onto a HOM by a CW beam.

\section{STATISTICAL ANALYSIS UNDER CW OPERATION}

\subsection{Preliminary discussion}

In order to know if a HOM coupler is needed, one has to estimate if the probability of occurrence of the "resonant case" is significant or not.

A first estimation of the deposited power can be given by considering the "theoretical case", assuming that the HOM characteristics (and in particular the frequency) are exactly the ones given by the RF codes. In the case of the 704.4 MHz $\beta=0.65$ 5-cell ASH cavity [3], taking into account all the 65 monopole and dipole modes (trapped or not) up to the $\mathrm{TM}_{01}$ cut-off frequency (at $2.6 \mathrm{GHz}$ ), and estimating the external Q's from the main power coupler only, one finds that the total power deposited by a 352.2 $\mathrm{MHz} 20 \mathrm{mACW}$ beam remains below a few tens of $\mathrm{mW}$.

But this theoretical case must be considered as an ideal case because, due to cavity manufacturing imperfections, the real resonant frequency of a HOM can never be exactly known before measuring it on the real cavity. Precise measurements performed on several $700 \mathrm{MHz}$ prototype cavities show differences of several $\mathrm{MHz}$ between the computed and measured frequencies [4]. Thus, one has to keep in mind that the very unlucky case where the frequency of all the most dangerous HOM exactly matches with a bunch resonance frequency has a non-zero probability. It can be showed that, in this "worst case", the power deposited in our $700 \mathrm{MHz}$ 5-cell cavity can theoretically exceed several MW!

\subsection{Statistical analysis}

A statistical method has been developed in order to compute the probability to deposit high powers onto the HOM of a SC cavity. This method is based on:

- Calculation of a HOM characteristics (especially its frequency $f_{\mathrm{HOM}}$ ) using RF codes like MAFIA or SUPERFISH.

- Accounting of the frequency spread due to manufacturing imperfections (gaussian distribution of the frequencies around $\mathrm{f}_{\mathrm{HOM}}$ with a standard deviation $\Sigma$ of a few MHz).

- Calculation of the probability to deposit more than $\mathrm{P}_{0}$ Watts onto the considered HOM. This probability is given by:

$$
\operatorname{Proba}\left(\mathrm{P}>\mathrm{P}_{0}\right)=\int_{\mathrm{f}_{0}-\Delta f\left(\mathrm{P}_{0}\right) / 2}^{\mathrm{f}_{0}+\Delta f\left(\mathrm{P}_{0}\right) / 2} \frac{1}{\sqrt{2 \pi} \Sigma} \mathrm{e}^{-\frac{\left(\mathrm{f}-\mathrm{f}_{\mathrm{HOM}}\right)^{2}}{2 \Sigma^{2}}} \cdot d f
$$

where $\Delta \mathrm{f}\left(\mathrm{P}_{0}\right)$ represents the bandwidth inside which $\mathrm{P}>\mathrm{P}_{0}$ (see Fig. 4).

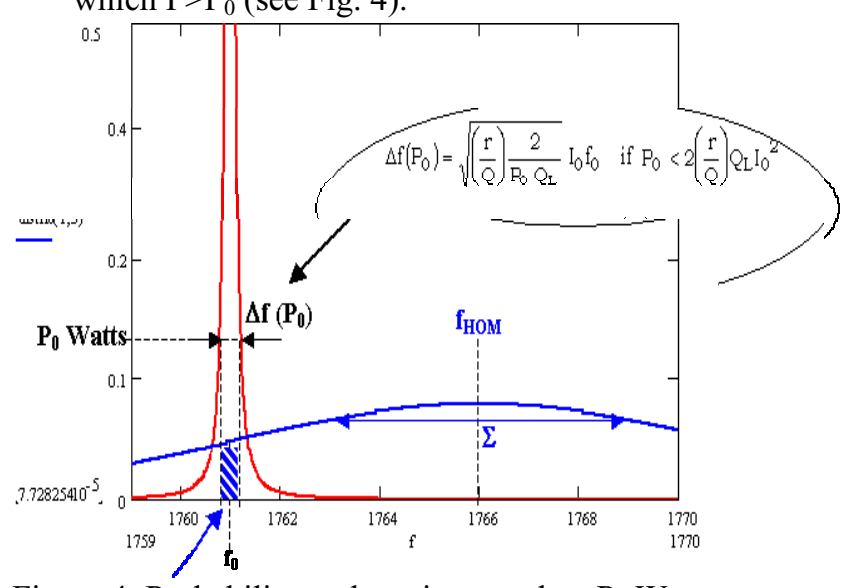

Figure 4: Probability to deposit more than $\mathrm{P}_{0}$ Watts on a HOM (streaked area); in red: power deposited by a CW beam; in blue: frequency distribution.

The final step consists in combining all the different HOM contributions (choosing the most relevant $\Sigma$ ) to obtain the total probability to deposit more than $\mathrm{P}_{0}$ Watts in the cavity. Fig. 5 represents the evolution of this probability as a function of $\mathrm{P}_{0}$ for different Q's values; these results show first that the probability to deposit high powers onto HOM is extremely low, and second that the use of specific HOM couplers (leading to lower Q-values) is not needed, and even not welcome since it leads generally to higher probabilities. 
Proceedings of the 2001 Particle Accelerator Conference, Chicago

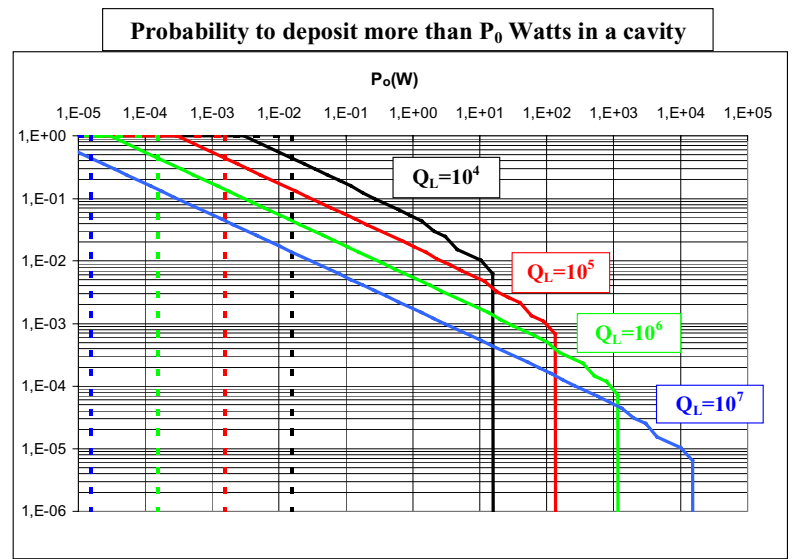

Figure 5: Probability to deposit more than $\mathrm{P}_{0}$ Watts in a 704.4 MHz 5-cell $\beta=0.65$ cavity as a function of $\mathrm{P}_{0}$ (all HOM Q's fixed at $10^{4}, 10^{5}, 10^{6}$ or $10^{7} ; 352.2 \mathrm{MHz}$ $20 \mathrm{~mA} 300 \mathrm{MeV} \mathrm{CW}$ beam; $\Sigma=10 \mathrm{MHz}$ in full line and $100 \mathrm{kHz}$ in dotted line)

Applying this method to our $700 \mathrm{MHz}$ cavities operating under a $20 \mathrm{~mA} \mathrm{CW}$ beam, and estimating the real external $\mathrm{Q}$ of each HOM from the main power coupler only (i.e. without HOM ports), it can be showed that the probability to deposit more than $1 \mathrm{~W}$ in a cavity is kept below $1 / 1000$ on the overall high energy section $(>100 \mathrm{MeV})$. This definitely confirms that specific HOM couplers are useless in such a $\mathrm{CW}$ superconducting linac. Note that this conclusion was also confirmed by cumulative beam break-up calculations showing that HOM excitation had no significant effect on both longitudinal and transverse beam stability [5].

\section{ABOUT PULSED OPERATION}

Following the analysis made for a $\mathrm{CW}$ beam, it is also possible to calculate the average power deposited onto a HOM by a pulsed beam (peak current: $\mathrm{I}_{\mathrm{p}}$; bunch period: $T_{b}$; bunch length: $\sigma_{b}$; pulse period: $T_{p}$; pulse length: $\sigma_{p}$ ). A careful analysis [6] shows that this average power takes the form:

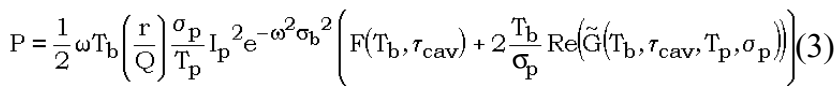

where $F\left(T_{b}, \tau_{\text {cav }}\right)$ is the CW component (see Eq. 1), while $\mathrm{G}\left(\mathrm{T}_{\mathrm{b}}, \tau_{\mathrm{cav}}, \mathrm{T}_{\mathrm{p}}, \sigma_{\mathrm{p}}\right)$ gives the pulse modulation $\left(\delta=\mathrm{T}_{\mathrm{p}}-\sigma_{\mathrm{p}}\right)$ :

$$
\tilde{G}\left(T_{b}, \tau_{c a v}, T_{p}, \sigma_{p}\right)=\frac{e^{j \omega T_{b}} e^{-T_{b} / \tau_{\text {cav }}}\left(1-e^{j \omega \sigma_{p}} e^{-\sigma_{p} / \tau_{\text {cav }}}\right)\left(e^{j \omega \delta} e^{-\delta / c_{\text {cav }}}-1\right)}{\left(1-e^{j \omega T_{b}} e^{-T_{b} / \tau_{\text {cav }}}\right)^{2}\left(1-e^{j \omega T_{p}} e^{-T_{p} / \tau_{\text {cav }}}\right)}
$$

(note that Eq. 3 must be divided by the duty cycle $\left(\sigma_{p} / T_{p}\right)$ to obtain the peak power deposited)

Analysing Eq. 3, it appears that the contribution of the pulse modulation leads in additional resonant build-ups every $1 / T_{p}$. This pulse contribution can be neglected if the Q-value is low enough, but it becomes preponderant when $\mathrm{Q}_{\mathrm{L}} \geq \omega \mathrm{T}_{\mathrm{p}} / 2$ - roughly (see Fig. 6). This especially means that the value of the pulse period is of great importance in this analysis: the shorter is the pulse period, the more the pulse resonances are dangerous (for a given Q-value).

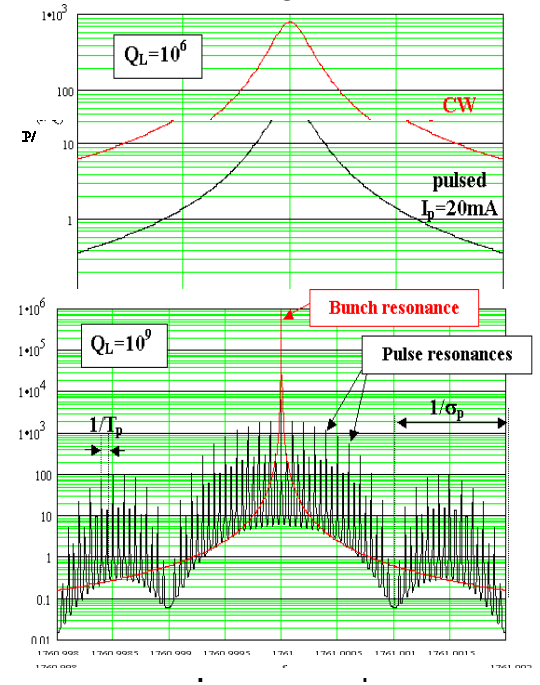

Figure 6: Average power deposited onto a HOM by a pulsed beam as a function of the HOM frequency: in red, case of a CW beam $(352.2 \mathrm{MHz}, 20 \mathrm{~mA})$; in black, case

of a pulsed beam $(352.2 \mathrm{MHz}, 20 \mathrm{~mA}$ peak, $50 \mathrm{~Hz}$

pulsing, $5 \%$ duty cycle); given for 2 different Q-values.

The statistical analysis presented in section 3 can be extended (using Eq. 3) to the case of a pulsed beam, allowing the calculation of the probability to deposit high HOM powers in a SC cavity under pulsed operation. This analysis can lead to various conclusions, according mainly to the beam structure (and especially the pulse period) and to the HOM characteristics of the considered cavity. A more detailed analysis of the HOM excitation by various pulsed beams will be reported elsewhere.

\section{CONCLUSION}

A statistical analysis is proposed for the study of the danger induced by HOM excitation in a SC linac. This analysis shows that the probability of occurrence of HOM build-up is extremely low under $\mathrm{CW}$ operation, leading to the conclusion that there is no need for specific HOM couplers in this case. Under pulsed operation, the situation is more complex, strongly depending on the beam structure.

\section{REFERENCES}

[1] H. Safa, "Superconducting Proton Linac for Waste Transmutation", Proc. of the $9^{\text {th }}$ Workshop on RF Superconductivity, Santa Fe, USA, Nov. 1999.

[2] E. Haebel, "Wakefields - resonant modes \& couplers", Frontiers of Accel. Technology, Proc. Joint US-CERNJapan Int. School, Hayama/Tsukuba, Japan, Sept. 1996.

[3] J-L. Biarrotte \& al, "704 MHz SC Cavities for a High Intensity Proton Accelerator", Proc. of the $9^{\text {th }}$ Workshop on RF Superconductivity, Santa Fe, USA, Nov. 1999.

[4] G. Olry, $5^{\text {th }}$ Meeting on SC Acceler. for Hybrid, CEA/INFN/IN2P3 collab., Orsay, France, Sept. 2000.

[5] N. Pichoff, A. Mosnier, private comm., Saclay, France, 2000.

[6] J-L. Biarrotte, PhD thesis, DAPNIA/SEA-00-01-T, Saclay, France, Jan. 2001. 